\title{
Remaining Useful Life estimation for informed End of Life Management of industrial assets: a conceptual model
}

\author{
Marco Macchi ${ }^{1}$, Irene Roda ${ }^{1}$ and Leonardo Toffoli ${ }^{1}$ \\ ${ }^{1}$ Department of Management, Economics and Industrial Engineering, Politecnico di Milano, \\ Milano, Italy \\ irene.roda@polimi.it
}

\begin{abstract}
The management of the End of Life phase of the lifecycle of industrial assets is more and more a relevant issue for companies dealing with aging assets. This paper provides a conceptual model that includes different aspects that should be considered by a comprehensive methodology to support an informed decisionmaking process in this regard. In particular, the work stems from the need of estimating the Remaining Useful Life (RUL) of an asset, for defining an Ageing Asset Strategy, through a multi-disciplinary approach instead of a purely technical one. To this end, the proposed model highlights the different End of Life types of an asset that should be considered for comprehensive RUL estimation methodology.
\end{abstract}

Keywords: Remaining Useful Life, Asset Management, Life Extension, End of Life.

\section{$1 \quad$ Introduction}

Nowadays, ageing assets represent a particular challenge for asset managers [1]. Society's inventory of capital goods is increasing as well as ageing in the western societies. This is very much the case for infrastructures like roads, railways, electric power generation, transport, and aircrafts [2] but also involves production facilities. Many manufacturing plants - at least in Europe - have been built in the years after the Second World War, and assets therein are currently approaching the end of their expected functional lives [3]. Ageing of asset systems is one of the reasons why physical Asset Management (AM) has become a more essential part of the organizations' activities during the last decades [4], [5]. Therefore, it is more and more important to define an effective Ageing Assets Strategy for companies managing capital assets. The implementation of such a strategy should provide asset managers with the tools they need to determine the most cost-effective strategy for the ageing assets under their stewardship [1].

As stated by [6], life extension is an alternative to conventional End of Life management strategies, such as decommissioning and replacement of capital equipment, and is gaining popularity in the last years. The decision of extending the life of an asset can potentially bring great economic advantages and value to asset owners, managers and stakeholders if all the necessary information is effectively collected to support it, and 
proper decision-making is applied. In fact, it is crucial for decision makers to have a holistic view on the current processes and issues involved in undertaking a life extension program.

Asset Life Extension processes generally include: definition of premises for the life extension program, assessment of asset condition, estimation of remaining useful life (RUL) and evaluation of different strategies for life extension [6]-[8].

The Asset Integrity Management (AIM) approach developed in the Oil\&Gas industry, can be considered the predecessor of the ageing and life extension programs that are nowadays under discussion. According to [7], AIM and Asset Life Extension are overlapping facets of the same requirement to ensure offshore safety, with the former principally concerned with contemporaneous integrity management, and the latter requiring a forward-looking approach anticipating future changes, challenges and threats, and forecasting the consequences on an installation's risk profile. In the document by [7], a process to preserve asset integrity and extend its life is proposed and its main phases are: to understand the asset condition, to recognize ageing and obsolescence, to manage life extension. Even if the model does not analyze in depth how condition assessment and RUL estimation should be performed, it gives an important remark: there are not only forces related to physical degradation at work in an ageing of asset but obsolescence should be taken into account too in industrial installations.

Based on these premises, this research aims at proposing a conceptual model to estimate the Remaining Useful Life (RUL) for informed-decision making in End of Life Management (EOL) of industrial assets, identifying and considering different potential causes of ageing, relevant in order to define an Asset Strategy through a multi-disciplinary approach instead of a pure technical one. Section 2 shows findings from the literature analysis about EOL causes and RUL estimation approaches. Section 3 describes the proposed conceptual model. Section 4 is dedicated to the Conclusions.

\section{$2 \quad$ Literature analysis}

Estimating the exact moment in which an asset would reach its EOL and therefore calculating its RUL is one essential step in the life extension process but it is not a trivial task [6]-[8]. RUL estimation is a key research topic of Condition Based Maintenance (CBM) and PHM ([9], [10]) but some gaps still exist, mainly related to the need of addressing the problem from a multi-disciplinary perspective and not only from a technical one [8]. In fact, one open issue in the literature is about ageing causes to be considered for RUL estimation in order to get to an indicator that can be used for informed AM decision-making, overcoming the pure physical ageing perspective of an asset.

For this reason, the first objective of this paper is to analyze and systematize the discussion about EOL causes. Secondly, the analysis of current approaches in literature for RUL estimation is presented. 


\subsection{End of Life Causes}

In the studies on life extension of assets in the Oil\& Gas industry [6], [7], the presence of two aspects when talking about ageing is hinted. Indeed, they suggest the separation between causes of ageing related to the physical condition of the asset and causes related to obsolescence, which can be linked to various factors other than physical degradation.

Other studies support this idea, by clearly stating the distinction of obsolescence from any type of physical degradation. For example, the work [11] demonstrated the presence of two forces of ageing with a case study distinguishing between what they call the traditional forces of mortality and the technology obsolescence. The work [12] recently confirmed this insight as they also asserted that the main reasons for the end of life of a system can be divided between physical ageing and wear, and obsolescence, that they define as the inability to satisfy increasing requirements of the users. Moreover, the authors identify three types of obsolescence, i.e., economic obsolescence, functional obsolescence and spare parts obsolescence, asserting that what other scholars have defined as technology obsolescence is one of the causes, possibly the most frequent, of the three obsolescence types.

Table 1 shows and classifies different types of EOL causes, adapting the work by [12] including other relevant literature.

Table 1. EOL causes classification

\begin{tabular}{|c|c|c|}
\hline EOL Causes & Type & Factors \\
\hline Physical Ageing & $\begin{array}{l}\text { Physical degradation (the asset is unable to } \\
\text { produce a predetermined quality or quantity } \\
\text { of output) }\end{array}$ & $\begin{array}{l}\text { Traditional ageing } \\
\text { mechanisms as wear } \\
\text { or corrosion }\end{array}$ \\
\hline \multirow{3}{*}{ Obsolescence } & $\begin{array}{l}\text { Economic Obsolescence (relative increase in } \\
\text { the costs of operations) }\end{array}$ & $\begin{array}{l}\text { Technology advance / } \\
\text { other causes }\end{array}$ \\
\hline & $\begin{array}{l}\text { Functional Obsolescence (the asset is unable } \\
\text { to satisfy new requirements) }\end{array}$ & $\begin{array}{l}\text { Changing needs of } \\
\text { client / introduction } \\
\text { of new regulations / } \\
\text { other causes }\end{array}$ \\
\hline & $\begin{array}{l}\text { Diminishing Manufacturing Sources and Ma- } \\
\text { terial Shortages (DMSMS) (difficulty in re- } \\
\text { pairing the asset) }\end{array}$ & $\begin{array}{l}\text { Spare parts unavaila- } \\
\text { bility / bankruptcy of } \\
\text { suppliers / other } \\
\text { causes }\end{array}$ \\
\hline
\end{tabular}

In detail, two main EOL causes can be defined: physical ageing and obsolescence. Physical ageing is considered as the main form of ageing by most of the literature, and its only cause is physical degradation increasing the failure risk. Most methodologies related to the RUL estimation are specifically dedicated to this type of EOL cause, as showed in the next section.

Obsolescence is the other EOL cause type and it can be classified into: 
- Economic obsolescence, caused mainly by technology advance and emerging when the current asset is no more profitable if compared with a new equipment;

- Functional obsolescence, emerging when the asset is unable to satisfy the new requirements after the introduction of new regulations or a change in the market;

- Diminishing Manufacturing Sources and Material Shortage (DMSMS) obsolescence, mainly related to the obsolescence of the spare parts leading to difficulty in repairing the asset caused, mostly but not only, by technology advance.

\subsection{Remaining Useful Life estimation approaches}

Currently, the estimation of the RUL is a topic of extensive research efforts [13]. Assuming the RUL would be known exactly, an asset could be exploited generating optimal value for its owner, without any increased failures or costs. Furthermore, knowing the processes or incidents that cause the end of the assets useful life would allow the owner to take preventive measures to extend the asset's life [3], [13]. However, there are some weaknesses related to current approaches to estimate asset RUL. In particular, [3] highlighted two of those weaknesses.

- many approaches are limited to the technical aspects of the asset or to a mainly statistical approach to deterioration mechanisms;

- many quantitative attempts fail because of the quality and availability of data.

Hence, on one side there is the need for methods that adopt a multi-disciplinary approach, considering other ageing causes together with physical degradation, connected with obsolescence [8]. On the other side, there is need for methods suitable for situations in which limited or no quantitative data are available, for example methods based on the knowledge of experts [10].

Looking at the RUL estimation techniques in the literature, it is clear that most scholars focused their studies mainly on the development of quantitative methods to support condition-based maintenance programs [14]-[19]. These works have been grouped and defined generically as quantitative RUL estimation methods and techniques because their objective is to link one or more degradation mechanisms to the life of the asset element, mainly low levels of the asset decomposition (in a complex asset structure), to predict when it will fail. They are often complex methodologies and models concentrating on a specific part or assembly to develop a technique that works in certain conditions. Besides, they can be very precise when data are available but they are difficult to be applied when reliable data is scarce. Moreover, as already stated, they are monodisciplinary since they are limited to the technical aspects of the asset. In [8], they recognized the importance for a multi-disciplinary approach for RUL prediction for Life Extension (LE) decision-making. Nevertheless, their model still focuses on the technical input to decision-making, by establishing a process for technical health assessment.

A group of scholars, starting from the difficulties that often emerge when using quantitative methodologies, tried to overcome them developing a model that considers also qualitative information along with quantitative data and that is multi-disciplinary [3]. In an attempt to make the RUL a multi-disciplinary practice, [3] propose a new 
methodology called Lifetime Impacts Identification Analysis that aims to identify the external impacts that could affect an asset's life in the future without trying to calculate the exact date of end of life. According to the authors it is important to consider four dimensions of ageing: i) the technological perspective, which is related to the question for how long the asset (and/or its output) will comply with the existing technical specifications, ii) the economic perspective, concerning the costs of operating and maintaining a piece of equipment, iii) the compliancy perspective, that deals with the 'license to operate' of the company, and iv) the commercial perspective, which considers whether the asset (and its production) are still able to fulfil the demands of the market. This methodology is an interesting starting point for research looking for comprehensive methodologies to support the EOL management of industrial assets.

\section{$3 \quad$ Proposed Conceptual Model}

Based on the analysis of the literature, the proposed conceptual model intends to provide the basis to develop a guide for asset managers to determine in a simple but systematic manner the RUL of an asset, enlarging the concept of RUL by considering different EOL causes together with the physical ageing. The proposed methodology is thought to facilitate a rigorous approach to address the problem, bearing in mind that each step of the process will require the application of systematic judgment and experience, to achieve informed decisions in the EOL phase and to eventually develop proper asset strategies.

The methodology is built on this simple postulate: while physical degradation is certainly causing the end of physical life, it is not the only way an asset can reach its EOL; starting from the definitions provided by the literature, different EOL types can be defined associated with different EOL causes. In particular, we define four types of EOL besides the end of physical life: i) end of service level life, ii) end of capacity life, iii) end of financial life, iv) end of maintainable life. In the reminder, each of it is described in detail and related to one type of EOL causes as defined in Table 1.

Regarding the physical ageing EOL cause, the EOL type can be identified as follows the EOL type can be identified as follows the EOL type can be identified as follows:

- End of physical life: it occurs when an asset is physically non-functioning (e.g., failed, collapsed, stopped working). Physical mortality failure occurs when the consumption of an asset caused by usage over time reduces performance to such an extent that the asset is unable to sustain performance at or above minimum requirements. A physical mortality failure could occur due to such things as age, wear and tear, environmental factors, accidental damage or operator error.

Regarding the Functional Obsolescence EOL cause, two types of EOL can be identified, related to external factors, and they are the following:

- End of service level life: it occurs when the expected levels of service have changed since the acquisition of the asset such that the performance requirements now imposed on the asset exceed the functional design capabilities of the asset. This could 
be due to changes in regulations (such as effluent, air, water quality or safety requirements) or due to changes in customer needs.

- End of capacity life: it occurs when the volume of demand placed on an asset exceeds its design capability.

Regarding the Economic Obsolescence EOL cause, one type of EOL can be identified, and it is:

- End of financial life: it occurs when an asset ceases to be the lowest cost alternative to satisfy a specified level of performance or service level, i.e. when the cost to sustain required performance from an asset under current O\&M practices exceeds that of feasible alternatives (where the amortized cost to acquire plus the costs to operate and maintain a new or renewed asset is less than the operation and maintenance of the existing asset). This type of EOL is often driven by outdated technology or design.

Regarding the DMSMS Obsolescence EOL cause, one type of EOL can be identified, and it is:

- End of maintainable life: it occurs when it becomes inconvenient to maintain the asset either because the costs of spare parts are increasing or because spare parts are not available in the market. This type of EOL is strictly connected with the unavailability of spare parts which can be caused by the development of a new technology, by a supplier's bankruptcy or by the supplier's decision, e.g. not to produce anymore the parts. This type of EOL is also connected with the End of financial life since it can be related to an increase in the costs of operating the asset as a consequence.

The different EOL types have to be considered to estimate RUL through a complete evaluation, and it is needed that the asset managers identify which type of EOL will likely rise first. In fact, while all five causes are at work on an asset at all times, only one type of EOL is expected to be the most imminent in time. By this perspective, RUL is defined as the lowest expected life for a selected asset given its operating environment where that life is derived from a determination of the most imminent EOL type, i.e. minimum value among the different types of Time to EOL (TT_x EOL):

$R U L=\operatorname{Min}\left(T T_{\text {physical EOL }}, T T_{\text {capacity EOL }}, T T_{\text {service level EOL }}, T T_{\text {financial EOL }}, T T_{\text {maintainable EOL }}\right)$

Once the RUL is estimated, it enables to forecast the point in time at which the asset will likely end its life. This is relevant for asset strategy at its EOL: it can be used to evaluate the lead time to be considered as the horizon to define the EOL strategy to adopt (e.g. major repair, refurbishment or replacement).

Figure 1 summarizes the steps to estimate the RUL of an asset following the proposed model. The most challenging aspect is the way to estimate each TT_x EOL since, for each type of EOL, many factors may incur (see Table 1) and should be considered to estimate it in a quantitative way. Moreover, uncertainty must be managed. Therefore, adequate sources of information and knowledge should be available, to finally obtain the most likely EOL of the asset. 
Fig. 1. Conceptual model for RUL estimation for informed decision-making

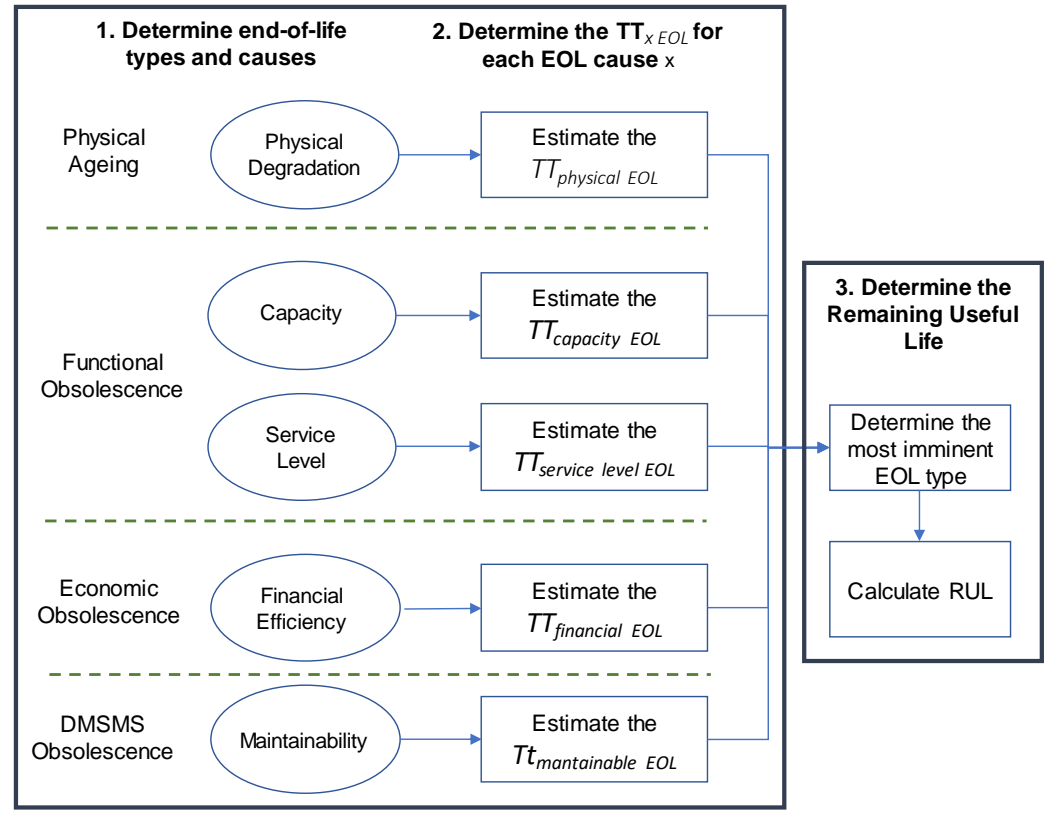

\section{Conclusions}

This paper discusses about the relevance of a comprehensive methodology to estimate the RUL of an industrial asset in order to support an informed decision-making process to manage its EOL phase. In fact, RUL estimation is a critical process for the definition of asset strategies at their EOL. Up to date, most RUL models that can be found in the literature focus on the physical ageing EOL cause. Very few attempts were made to estimate the impact of obsolescence on the assets EOL from a multi-disciplinary perspective. In this paper, different EOL causes are identified and classified and, based on that, a conceptual model is proposed including the EOL types that should be considered in a comprehensive methodology to be used for Asset Management decision making about the EOL of industrial assets. The proposed model can be used as a reference for future research on methods for decision and information support in EOL management of industrial assets. The model is intended to be conceptual and opens the path for future research on the methods that can be used for transferring the various aspects of End of life to Time to EOL and hence, to estimate the RUL through the proposed perspective. In fact, different methods can fit this model, either semi-quantitative like multi-criteria decision making supporting methods, either quantitative like the use of optimization based on consideration of optimal usage of the asset. The proposed model can be also useful in the context of Product-Service Systems both for the asset owner/operator and the OEMs [20] and future research on this aspect is envisioned by the authors. 


\section{References}

1. IAM: Asset Management - an anatomy (2012).

2. Budai G., Dekker R., and Nicolai R. P.: Maintenance and Production: A Review of Planning Models. Complex System Maintenance Handbook, London: Springer London, 321-344, (2008).

3. Ruitenburg R. J., Braaksma A. J. J., and Van Dongen L. A. M.: A multidisciplinary, expertbased approach for the identification of lifetime impacts in Asset Life Cycle Management. Procedia CIRP, 22(1), 204-212 (2014).

4. CSN EN 16646: Maintenance within physical asset management. European Standard (2014).

5. Amadi-Echendu J. E. and Brown K.: Definitions, Concepts and Scope of Engineering Asset Management (2010).

6. Shafiee M. and Animah I.: Life extension decision making of safety critical systems: An overview. J. Loss Prev. Process Ind., 47, 174-188 (2017).

7. Oil\&Gas-UK: Guidance on the Management of Ageing and Life Extension for UKCS Oil and Gas Installations, 1 (2012).

8. Vaidya P. and Rausand M.: Remaining useful life, technical health, and life extension. Proc. Inst. Mech. Eng. Part O J. Risk Reliab., 225(2), 219-231 (2011).

9. Jardine A. K. S. and Tsang A. H. C., Maintenance, replacement and reliability: theory and applications, Boca Raton (2006).

10. Si X.-S., Wang W., Hu C.-H., and Zhou D.-H.: Remaining useful life estimation - A review on the statistical data driven approaches, Eur. J. Oper. Res., 213(1), 1-14, (2011).

11. Barreca S. L. and Kateregga K. A.: Technology Life-Cycles And Technological Obsolescence. BCRI Inc (2000).

12. Lomakin M. I. and Murav'ev A. V.: Managing the Process of Re-Engineering of Information Systems Based on Integrated Monitoring of Obsolescence, Meas. Tech., 58(10), 1102-1106 (2016).

13. Guillén A. J., Crespo A., Macchi M., and Gómez J.: On the role of Prognostics and Health Management in advanced maintenance systems. Prod. Plan. Control, 27(12), 991-1004 (2016).

14. Sobral J. and Guedes Soares C.: Preventive Maintenance of Critical Assets based on Degradation Mechanisms and Failure Forecast. IFAC-PapersOnLine, 49(28), 97-102 (2016).

15. Chen X., Yu J., Tang D., and Wang Y.: Remaining useful life prognostic estimation for aircraft subsystems or components: A review. IEEE 2011 10th International Conference on Electronic Measurement \& Instruments, pp. 94-98 (2011).

16. Okoh C., Roy R., Mehnen J., and Redding L.: Overview of Remaining Useful Life Prediction Techniques in Through-life Engineering Services. Procedia CIRP, 16, 158-163 (2014).

17. Animah I. and Shafiee M.: Condition assessment, remaining useful life prediction and life extension decision making for offshore oil and gas assets. J. Loss Prev. Process Ind., 1-12 (2016).

18. Wang H.-K., Haynes R., Huang H.-Z., Dong L., and Atluri S. N.: The Use of High-Performance Fatigue Mechanics and the Extended Kalman / Particle Filters, for Diagnostics and Prognostics of Aircraft Structures. C. Comput. Model. Eng. Sci., 105(1), 1-24 (2015).

19. Wang X., Rabiei M., Hurtado J., Modarres M., and Hoffman P.: A probabilistic-based airframe integrity management model. Reliab. Eng. Syst. Saf., 94(5), 932-941 (2009).

20. West, S. \& Wuest, T.: A Strategy for Midlife Upgrades to Provide Value for Both the Equipment Operator and the Supplier. Spring Servitization Conference (SSC 2017), (2017). 